\title{
Disconnection of the Perirhinal and Postrhinal Cortices Impairs Recognition of Objects in Context But Not Contextual Fear Conditioning
}

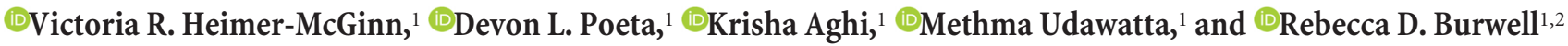 \\ 'Department of Cognitive, Linguistic, and Psychological Sciences and 'Department of Neuroscience, Brown University, Providence, Rhode Island 02912
}

The perirhinal cortex (PER) is known to process object information, whereas the rodent postrhinal cortex (POR), homolog to the parahippocampal cortex in primates, is thought to process spatial information. A number of studies, however, provide evidence that both areas are involved in processing contextual information. In this study, we tested the hypothesis that the rat POR relies on object information received from the PER to form complex representations of context. Using three fear-conditioning (FC) paradigms (signaled, unsignaled, and renewal) and two context-guided object recognition tasks (with 3D and 2D objects), we examined the effects of crossed excitotoxic lesions to the POR and the contralateral PER. Performance of rats with crossed lesions was compared with that of rats with ipsilateral POR plus PER lesions and sham-operated rats. We found that rats with contralateral PER-POR lesions were impaired in object-context recognition but not in contextual FC. Therefore, interaction between the POR and PER is necessary for context-guided exploratory behavior but not for associating fear with context. Our results provide evidence for the hypothesis that the POR relies on object and pattern information from the PER to encode representations of context. The association of fear with a context, however, may be supported by alternate cortical and/or subcortical pathways when PER-POR interaction is not available. Our results suggest that contextual FC may represent a special case of context-guided behavior.

Key words: hippocampus; lesion; medial temporal lobe; parahippocampal; recognition memory; spatial

Significance Statement

Representations of context are important for perception, memory, decision making, and other cognitive processes. Moreover, there is extensive evidence that the use of contextual representations to guide appropriate behavior is disrupted in neuropsychiatric and neurological disorders including developmental disorders, schizophrenia, affective disorders, and Alzheimer's disease. Many of these disorders are accompanied by changes in parahippocampal and hippocampal structures. Understanding how context is represented in the brain and how parahippocampal structures are involved will enhance our understanding and treatment of the cognitive and behavioral symptoms associated with neurological disorders and neuropsychiatric disease.

\section{Introduction}

Contextual representations are important for many cognitive functions, including episodic memory. Although the medial temporal lobe is implicated in contextual learning, how and where context is represented is still under debate. Much of the literature

\footnotetext{
Received Jan. 26, 2017; revised March 28, 2017; accepted April 2, 2017.

Author contributions: D.L.P. and R.D.B. designed research; V.R.H.-M., D.L.P., K.A., M.U., and R.D.B. performed research; V.R.H.-M., D.L.P., and R.D.B. analyzed data; V.R.H.-M. and R.D.B. wrote the paper.

This work was supported by the National Science Foundation (Grant IOB-0522220 to R.D.B.) and the National Institutes of Health (Grant 5T32MH019118 to V.R.H.-M. and Grant 5F32MH105210 to V.H.M.).

The authors declare no competing financial interests.

Corresponding author: Rebecca D. Burwell, Ph.D., Department of Cognitive, Linguistic, and Psychological Sciences, Brown University, 190 Thayer Street, Box 1821, Providence, RI 02912. E-mail: rebecca_burwell@brown.edu. https://doi.org/10.1523/JNEUROSCI.0254-17.2017

Copyright $\odot 2017$ the authors
}

focuses on the modularity of the two parallel-processing streams, spatial and nonspatial, that converge on the hippocampus (Burwell, 2000; Eichenbaum et al., 2007; Eichenbaum et al., 2012). In this view, the hippocampus receives spatial information from the postrhinal cortex (POR) via the medial entorhinal cortex and nonspatial information from the perirhinal cortex (PER) via the lateral entorhinal cortex and then binds these two streams of information to represent context. Substantial anatomical evidence, however, reveals functional integration across these two pathways and indicates that representations of context may be formed in the POR upstream of the hippocampus (Suzuki and Amaral, 1994; Burwell and Amaral, 1998; Dolorfo and Amaral, 1998; Chrobak and Amaral, 2007).

The role of the POR and its primate homolog, the parahippocampal cortex (PHC), may not be limited to processing infor- 


A Surgery Unsignaled FC 3D stdSOR
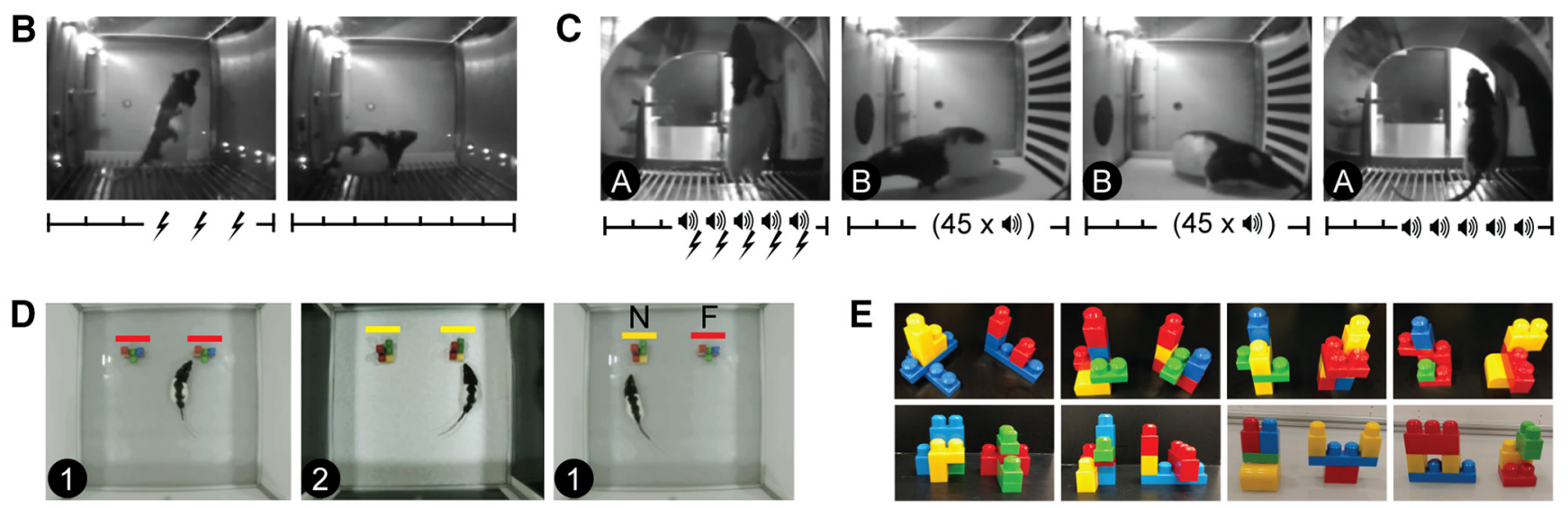

Figure 1. Design for Experiment 1. A, Timeline for procedures and tasks. $\boldsymbol{B}$, In the unsignaled FC paradigm, rats received three unsignaled foot shocks on the training day, followed by context extinction on the second day. C, FC ABBA renewal paradigm consisted of 5 tone-shock pairings in context $A$ on the training day followed by $2 \mathrm{~d}$ of tone extinction. On the fourth day, rats were returned to context $A$ for the renewal test. For $\boldsymbol{B}$ and $\boldsymbol{C}$, the $x$-axis shows timing of shocks (lightning icon) and tones (speaker icon). Tick marks represent 1 min post-tone blocks and " $45 \times$ speaker" indicates that 45 tones were presented with 30 s intertrial intervals. $\boldsymbol{D}$, Context-guided spontaneous object recognition task consisted of two sample phases in contexts 1 and 2 followed by a test phase in context 1. During the test (right), one object was novel in that particular context (N, yellow bar) and the other was familiar (F, red bar). E, 3D objects were made of Mega Bloks. A new pair was used for each run (8 runs, $48 \mathrm{~h}$ apart).

mation about space; rather, it may have a particular role in representing the spatial layout of objects, features, and patterns in the local context. Several lines of evidence implicate the POR/ $\mathrm{PHC}$ in the formation of contextual representations, a function that requires visual and spatial information processing (Gabrieli et al., 1997; Maguire et al., 1998; Burgess et al., 2001; Ranganath et al., 2004; Hayes et al., 2007). Human imaging studies suggest that the PHC processes objects with strong contextual associations (Bar and Aminoff, 2003) and objects that are considered space defining (Mullally and Maguire, 2011; Martin et al., 2013) and that the PHC is activated preferentially when viewing pictures of scenes, maps, and landmarks (for review, see Aminoff et al., 2013). Patients with PHC damage show deficits in navigation, spatial orientation, landmark identification, and spatial memory (Aguirre and D'Esposito, 1999; Ploner et al., 2000). Monkeys with PHC damage show impaired object location and object context recognition (Malkova and Mishkin, 2003; Bachevalier et al., 2015). Finally, POR neurons in rats show conjunctive coding of specific objects in particular places (Furtak et al., 2012). These findings are consistent with the idea that the POR/PHC may play a role in representing the spatial layout of objects in the local environment.

If the POR indeed encodes representations of context, then it must receive information about objects located there. The most obvious source of such information is the PER. In support of this view, an extensive body of anatomical data in rats and monkeys show strong and reciprocal direct connections between the PER and POR, suggesting a site of functional integration across the spatial and nonspatial streams (Suzuki and Amaral, 1994; Burwell and Amaral, 1998). Experimental lesion studies in rodents further show that bilateral damage to either the POR or PER disrupts contextual fear conditioning (FC) (Corodimas and LeDoux, 1995; Bucci et al., 2000; Burwell et al., 2004), context discrimination (Bucci et al., 2002), scene discrimination (Gaffan et al., 2004), and contextguided object recognition (Norman and Eacott, 2005). We posit that these impairments arise because the PER-POR connection is essential for the formation of contextual representations. More specifi- cally, we propose that projections from the PER deliver object information directly to the POR. The POR, then, forms representations of environmental context including the spatial layout of objects and features in the local environment.

To test the hypothesis that the POR and PER interact to form representations that bind objects, patterns, and other environmental features to represent context, we used a disconnection approach in rats. Rats with crossed PER-POR lesions were tested comprehensively for impairments in contextual FC and object context recognition. In Experiment 1, we used two FC paradigms and a context-guided spontaneous $3 \mathrm{D}$ object recognition (SOR) task. In Experiment 2, we used a different conditioning paradigm and a $2 \mathrm{D}$ version of the context SOR to replicate and extend the findings from Experiment 1. Deficits on both types of tasks caused by contralateral PER-POR lesions would imply that all context-guided behaviors rely on communication between these two structures. We found, however, that PER-POR disconnection impaired context-guided spontaneous exploration of objects, but not contextual FC.

\section{Materials and Methods}

Subjects

We used 18 adult male Long-Evan rats for Experiment 1 and 28 adult male rats for Experiment 2 (Charles River Laboratories). Of the 28 rats in Experiment 2, six were eliminated due to husbandry-related issues, leaving a total of 22 subjects. Housing, handling, and age/weight at the time of surgery were identical to Experiment 1 . On arrival, all rats were pair housed for $10 \mathrm{~d}$ in diurnal conditions ( $12 \mathrm{~h}$ light/dark cycle) with ad libitum access to food and water. They were then separated into individually ventilated cages and maintained at $85-90 \%$ body weight. Rats were handled at least five times before surgery. At the time of surgery, all subjects were 3-5 months old and weighed $250-300 \mathrm{~g}$. All testing was performed during the light phase. After recovery from surgery, rats were handled at least three times before the start of each experiment. The timeline for the two experiments is shown in Figures $1 A$ and $2 A$.

These experiments were performed in accordance with National Institutes of Health's guidelines for the care and use of rats in research. The 
A

\section{Surgery Signaled FC 2D cxtSOR}

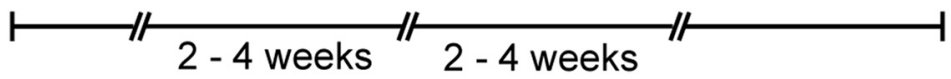

B

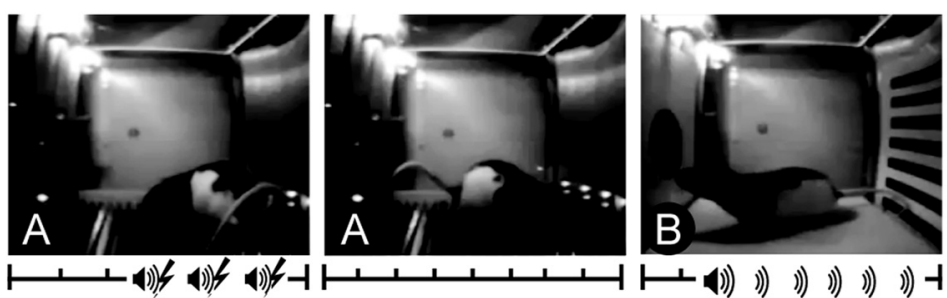

C

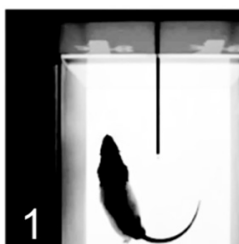

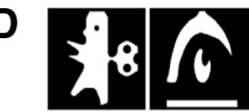

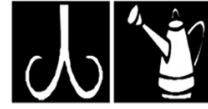

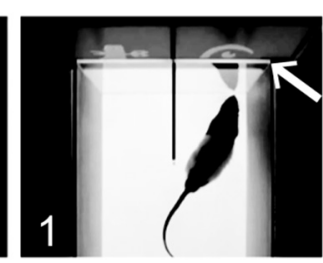
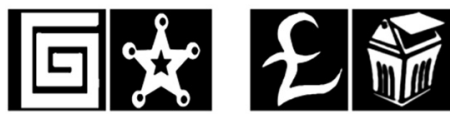

Figure 2. Design for Experiment 2. A, Timeline for procedures and tasks. B, Signaled FC paradigm consisted of training to three tone-shock pairings on the training day in context $A$, context extinction on day 2 in context $A$, and tone extinction on day 3 in context B. Axes show timing of tones (speaker icon), shocks (lightning icon), and 1 min blocks (tick marks). C, 2D cxtSOR task consisted of two sample phases in context 1 and context 2 followed by a test phase in which both objects are presented in context 1. The arrow indicates the novel object- context pairing. Context was operationalized as floor pattern only. $2 \mathrm{D}$ object images were presented vertically on a monitor. D, Clipart images served as 2D objects; a new pair was used for each run ( 4 runs, $24 \mathrm{~h}$ apart).

(ML) coordinates were calculated relative to bregma. Dorsal to ventral (DV) coordinates were calculated relative to the top of the skull. For POR lesions, lambda was measured at an angle of $16^{\circ}$ from vertical in the coronal plane and four sites were targeted (Table 1). AP and ML coordinates were calculated relative to lambda and DV coordinates relative to the top of the skull. Neurotoxic lesions were made using NMDA (Tocris Bioscience). For Experiment 1 , NMDA $(0.09 \mathrm{M}$ in $0.1 \mathrm{M}$ phosphate buffer) was delivered by pressure injection at $0.1 \mu \mathrm{l} / \mathrm{min}$ for $1 \mathrm{~min}$ at each site. The pipette was left in place for $3 \mathrm{~min}$ after each injection and then slowly retracted. For Experiment 2, NMDA ( $250 \mu \mathrm{M}$ in $0.5 \mathrm{~N}$ sodium hydroxide) was delivered iontophoretically using continuous direct current $(-6 \mu \mathrm{A})$ for $10 \mathrm{~min}$ at each site. The pipette was left in place for $1 \mathrm{~min}$ after each injection and then retracted slowly. For both experiments pipette tips were $45-50 \mu \mathrm{m}$. For sham surgeries, an empty glass pipette was lowered to $1 \mathrm{~mm}$ above the target sites and left in place for $30 \mathrm{~s}$. For all surgeries, the skin was sutured and rats were allowed to recover for 1 week before handling and for an additional week before behavioral testing.

\section{Apparatus}

FC tasks. Testing was performed in a dedicated room that houses four behavioral chambers $(21.6 \times 17.8 \times 12.7 \mathrm{~cm}$; Med Associates $)$. Each chamber had two aluminum side panels and

Plexiglas front door, rear wall, and ceiling.

Table 1. PER and POR lesion coordinates

\begin{tabular}{lllll}
\hline Site & Angle & AP & ML & DV \\
\hline PER 1 & $13^{\circ}$ & 2.8 & 5.0 & 6.0 \\
PER 2 & $13^{\circ}$ & 3.9 & 5.0 & 6.0 \\
PER 3 & $13^{\circ}$ & 5.0 & 5.0 & 5.8 \\
PER 4 & $13^{\circ}$ & 6.1 & 5.0 & 5.4 \\
PER 5 & $13^{\circ}$ & 7.2 & 5.0 & 5.0 \\
POR 1 & $16^{\circ}$ & 0.5 & 4.6 & 5.0 \\
POR 2 & $16^{\circ}$ & 0.5 & 4.6 & 4.0 \\
POR 3 & $16^{\circ}$ & -0.2 & 4.3 & 3.0 \\
POR 4 & $16^{\circ}$ & -0.2 & 4.3 & 2.5 \\
\hline
\end{tabular}

AP and ML coordinates for the PER and POR were measured relative to bregma and lambda, respectively. Angles are degrees from vertical in the coronal plane with tip directed laterally. DV coordinates were measured relative to the skull.

protocol covering these experiments was approved by the Brown University Institutional Animal Care and Use Committee.

\section{Surgery}

Rats were assigned randomly to one of the three lesion groups and lesion sides were counterbalanced within each group. For Experiment 1 , six rats received contralateral lesions to the PER and POR, six received ipsilateral lesions to the PER and POR, and six received sham lesions to the PER and POR (either ipsilaterally or contralaterally). For Experiment 2, seven rats received contralateral lesions to the PER and POR, seven received ipsilateral lesions to the PER and POR, and eight received sham lesions to the PER and POR (either ipsilaterally or contralaterally). Rats were anesthetized with isoflurane and secured in a stereotaxic frame. The incisor bar was adjusted such that the bregma and lambda were in the same horizontal plane $( \pm 0.2 \mathrm{~mm})$. Craniotomies were made using a dental drill and the dura removed to allow insertion of the glass pipette into the target brain region. For PER lesions, bregma was measured using the pipette at an angle of $13^{\circ}$ from vertical in the coronal plane and five sites were targeted (Table 1). Anterior to posterior (AP) and medial to lateral
Each floor consisted of evenly spaced stainless steel rods attached to a shock generator and scrambler for the delivery of a foot shock. A house light $(28 \mathrm{~V} ; 100 \mathrm{~mA}$ ) was located on the left side panel (centered; $10 \mathrm{~cm}$ from the top). Each chamber was enclosed in a $62 \times 56 \times 56 \mathrm{~cm}$ soundattenuating cabinet fitted with an exhaust fan that provided air flow to the test chamber and background noise. A computer-automated sound generator was interfaced with the system to provide programmable auditory stimuli. A video camera was mounted on the back wall of each soundattenuating chamber and used to record behavior in all four chambers simultaneously. The apparatus was controlled by Med-PC programs (Med Associates).

For the unsignaled FC, the conditioning chamber was used without alteration (Fig. $1 B$ ). For the ABBA renewal paradigm, contexts A and $\mathrm{B}$ were differentiated using visual, tactile, olfactory, auditory, spatial, and extramaze cues (Fig. 1C). Context A was as follows: sturdy black lamination bent to make the chamber appear arch shaped, house lights off, cabinet door open, room lights on, vanilla scent in chamber tray, house fan off, and rats transported in their home cages. Context B was as follows: black/white striped lamination on left wall, white lamination with large black dot on right wall, white acrylic slab placed over grid floor, house lights on, cabinet door closed, room lights off, isopropyl alcohol in chamber tray, house fan on (for noise), and rats transported in a plastic container. For signaled FC, contexts A and B were differentiated by visual, tactile, and olfactory cues (Fig. $2 B$ ). In context A, the behavioral chamber was unmodified. In context $\mathrm{B}$, the walls of the chamber were decorated with laminations of stripes (left wall) and a large black dot (right wall), a white acrylic slab was placed over the grid floor, and vanilla scent was added to the chamber tray.

Object recognition tasks. For the $3 \mathrm{D}$ object recognition tasks, testing was performed in a bottomless square arena $(80 \mathrm{~W} \times 80 \mathrm{D} \times 60 \mathrm{H} \mathrm{cm})$ and placed over a white acrylic table. All four walls were made of white matte acrylic to avoid multiple image reflections. The apparatus was housed in an isolated behavior room monitored by an overhead video camera (room lights on). The camera was interfaced with a computer in a separate room that displayed and recorded live footage. For the stan- 
dard object recognition task, the white box was placed on the smooth white glossy acrylic surface of the table. For the context-guided SOR, contexts 1 and 2 were differentiated using visual, tactile, and olfactory cues (Fig. 1D). In context 1 , the white box was placed on the smooth white glossy acrylic surface of the table. In context 2 , the walls were lined with black laminations (lightly sanded) and the floor was covered with a white stone-patterned rubber bath mat (Target); the distinct smell of rubber provided the olfactory cue. Objects (Fig. $1 E$ ) measured up to $12 \mathrm{~cm}$ in each dimension and were constructed using Mega Bloks (Mega Brands) with Blu-Tack (Bostik Australia). During each phase of the two tasks, two objects were placed $25 \mathrm{~cm}$ from the back wall with $\sim 35 \mathrm{~cm}$ between them (measured from the center of the object).

For 2D context-guided object recognition, testing was performed in a rectangular arena $(40 \mathrm{~W} \times 30 \mathrm{D} \times 40 \mathrm{H} \mathrm{cm}$; topless and bottomless) made of white matte acrylic placed on a floor projection maze (Jacobson et al., 2014). In this apparatus, a "short-throw" projector is used to backproject images/patterns onto a transparent acrylic tabletop overlaying a white projection screen. The floor projection maze was housed in an isolated behavior room monitored by an overhead video camera and interfaced to two computers in a separate room; these computers were used to control automated projections and display, record, and score the live footage. For context 1, a solid gray image was back-projected onto the floor; for context 2, a striped gray floor was back projected (Fig. 2C). The front portion of the arena was divided into two equal compartments using an opaque wall that extended $15 \mathrm{~cm}$ into the front arena and $4.5 \mathrm{~cm}$ beyond the front wall. A vertical computer monitor (Epson) abutted the $4.5 \mathrm{~cm}$ extension wall and was used to display objects. The front wall was made of transparent acrylic to allow viewing of the monitor. Black-andwhite 2D clipart images (Fig. 2D) were obtained online from Microsoft Word and scaled to measure $\sim 10 \times 15 \mathrm{~cm}$ onscreen. During the task, two objects were presented simultaneously on the computer screen such that one object was displayed in the front center of each compartment in the arena. Testing was performed in the dark to enhance image contrast.

\section{Behavioral procedures}

Unsignaled contextual FC. On training day, the rats were placed in individual chambers (Fig. $1 B$ ) and, after 3 min, received 3 constant-current shocks ( $1 \mathrm{~s}, 1.0 \mathrm{~mA}, 1 \mathrm{~min}$ intertrial interval). Twenty-four hours later, rats were returned to the same conditioning chambers for a $7 \mathrm{~min}$ extinction test, during which no shocks were delivered. The procedure was performed in cohorts of three (one rat per lesion group) and chamber placement was counterbalanced for lesion group across cohorts. Behavioral responses were videotaped for subsequent analysis.

Tone-signaled $A B B A$ and renewal FC. For nine rats (three from each group), training was performed in context $\mathrm{A}$; rats were assigned to an individual chamber for all phases of the task (Fig. 1C). On daily trials, rats were placed inside the operant chamber. After $3 \mathrm{~min}, 5$ tones $(10 \mathrm{~s}, 2 \mathrm{kHz}$, $80 \mathrm{~dB})$ that coterminated with a foot shock ( $1 \mathrm{~s}, 1.0 \mathrm{~mA})$ were delivered, each followed by a 1 min post-shock interval. On the following $2 \mathrm{~d}$, the rats were extinguished in context B; after 3 min, 45 tones were delivered, each followed by a $30 \mathrm{~s}$ post-tone period. On the fourth day, the renewal was tested in context A; after 3 min, 5 tones (but no foot shocks) were delivered, each followed by a $1 \mathrm{~min}$ post-tone period. The other nine rats experienced contexts BAAB. Test context was counterbalanced for lesion group. Chamber placement counterbalancing and videotaping were identical to the unsignaled paradigm.

signaled FC. Training was performed in context A. Rats were placed in the operant chamber (Fig. $2 B$ ). After $3 \mathrm{~min}, 3$ tones $(10 \mathrm{~s}, 2 \mathrm{kHz}, 80 \mathrm{~dB})$ that coterminated with a foot shock $(1 \mathrm{~s}, 1.0 \mathrm{~mA})$ were delivered, each followed by a $1 \mathrm{~min}$ post-shock epoch. On the second day, rats were extinguished to context A for $8 \mathrm{~min}$ (no tone). On the third day, they were extinguished to tone in context $\mathrm{B}$. Two minutes after rats were placed in the chamber, a tone was delivered continuously for 6 min, followed by a $1 \mathrm{~min}$ rest period. Counterbalancing and videotaping were identical to the unsignaled FC.

Context-dependent spontaneous $3 \mathrm{D}$ object recognition (3D cxtSOR). The task consisted of two sample phases followed by a test phase (Fig. $1 D)$. In sample phase $1(\mathrm{~S} 1)$, two identical objects (AA) were presented in context 1 . In sample phase 2 (S2), two new identical objects (BB) were presented in context 2 . In the test phase $(\mathrm{T})$, one of the two contexts was presented and a new copy of each object $(\mathrm{AB})$ was presented. The rat remained in the arena for $30 \mathrm{~s}$ of active object exploration or $5 \mathrm{~min}$, whichever came first. In between each phase, subjects were returned to their home cage for a $5 \mathrm{~min}$ delay period. Five minutes were chosen in accordance with Norman and Eacott (2005), who found that rats with either PER or POR lesions are impaired at this delay. Eight runs were performed with at least $48 \mathrm{~h}$ between runs and using new object pairs for each run (Fig. 1E). Context in the test phase, recency of the test context, and recency and side of the object in the novel object-context pair were counterbalanced across runs. Object and recency of the object in the novel object-context pair were counterbalanced across rats. Before behavioral testing, rats received 6 habituation sessions on 6 consecutive days; twice to the apparatus, once to each context, and once to each context with two identical objects. Order of contexts experienced was counterbalanced across rats and objects used for habituation were not reused in the task.

Standard spontaneous $3 \mathrm{D}$ object recognition (3D stdSOR). The procedure for this task was similar to the 3D cxtSOR except that there was only one sample phase (S) and one test phase (T). Two identical objects (AA) were presented in the sample phase. In the test phase, one object was replaced with a novel object (AB). The same context (white walls and acrylic floor) was used for sample and test phases. Side of novel object was counterbalanced across runs and the object used as the novel object was counterbalanced across rats (within each run).

Context-dependent spontaneous $2 \mathrm{D}$ object recognition ( $2 \mathrm{D}$ cxtSOR). Rats were placed in an "empty" arena and 2D images were presented after a 10-20 s delay (Fig. 2C). Subjects remained in the arena for $15 \mathrm{~s}$ of active exploration or $5 \mathrm{~min}$ from image onset, whichever came first. Exploration was recorded when the rat's nose was inside the left or right compartment (past the divider) pointing toward the image and the rat was not grooming, rearing, or interacting with the divider. The task was repeated daily for 4 consecutive days. All other conditions (e.g., habituation, task structure, counterbalancing, expected outcome) were identical to the 3D cxtSOR procedures.

\section{Behavioral analysis}

FC tasks. Videos were recorded using EthoVisionXT 9 (Noldus Information Technology) and freezing behavior was scored using EthoVision XT 11. Accurate automated scoring was verified manually by an investigator who was blinded to lesion group. Percentages of total freezing times were then calculated in blocks of $60 \mathrm{~s}$ (unless otherwise noted). For unsignaled FC, on training day, freezing was recorded and analyzed during $3 \mathrm{~min}$ before foot shock delivery and during 3 post-shock epochs, for a total of 3 baseline blocks and 3 post-shock blocks. For context extinction, a $7 \mathrm{~min}$ period was divided evenly into 7 blocks. For renewal FC on training day, freezing was recorded during $3 \mathrm{~min}$ before tone/foot shock delivery and during 5 post-shock epochs, for a total of 3 baseline blocks and 5 postshock blocks. For extinction, freezing was recorded during 3 min before tone delivery and during 45 post-tone epochs, for a total of 3 baseline blocks ( $60 \mathrm{~s}$ each) and 9 post-tone blocks ( $150 \mathrm{~s}$ each; sum of 5 consecutive $30 \mathrm{~s}$ periods). For renewal, blocks were identical to training. For signaled FC, analyses were identical to the unsignaled FC except that context extinction was divided into 860 s blocks rather than 7 and tone extinction was divided into 2 baseline blocks and 6 post-tone blocks.

Object recognition tasks. Exploration times were scored manually using Med-PC IV software (Med Associates) to control a button box interfaced with SmartCTL Interface Module (DIG-716B; Med Associates). Exploration was recorded when the rat's nose was within $4 \mathrm{~cm}$ of the object and pointed toward it, and the rat was not grooming, rearing, or interacting with the object (e.g., biting, licking, walking over it). Experimenters were blind to lesion group. Time stamps for start and end of exploration bouts were recorded in a Med Associates data file and extracted using a custom MATLAB script (The MathWorks). The primary measure of object recognition was a discrimination ratio (DR) constructed from exploration times of the novel (N) and familiar (F) object-in-context during the test phase. The DR measures the difference in exploration times for each object and expresses it as a proportion of the total exploration time; $\mathrm{DR}=(\mathrm{N}-\mathrm{F}) /(\mathrm{N}+\mathrm{F})($ Ennaceur and Delacour, 1988). A positive DR 
indicates preferential exploration of the object that is novel in a given context. In addition to DR, we also analyzed the frequency and duration of exploration bouts to determine whether exploratory behavior was different across groups. We were especially interested in bout duration because novel object exploration is often accompanied by increased duration of exploratory bouts compared with exploration of familiar objects (Renner and Seltzer, 1991). We also assessed bout number because, in other studies, we have found that increased exploration time can be accounted for by increased bout duration, increased bout number, or both (Ho et al., 2015). Consistent with previous studies (Norman and Eacott, 2005; Wilson et al., 2013), only data acquired in the first 3 min of exploration were used in the analyses. For the $2 \mathrm{D}$ cxtSOR, analyses were identical to the $3 \mathrm{D}$ cxtSOR.

\section{Histology}

Rats were deeply anesthetized with an intraperitoneal injection of sodium pentobarbital (Beuthanasia-D; Schering-Plough) and perfused transcardially at a rate of $35-40 \mathrm{ml} / \mathrm{min}$, first with saline and then with $10 \%(\mathrm{w} / \mathrm{v})$ formalin in $0.1 \mathrm{M} \mathrm{PB}$. Each brain was extracted, postfixed for at least $24 \mathrm{~h}$ at $4^{\circ} \mathrm{C}$ in the same solution, and cryoprotected for at least $48 \mathrm{~h}$ with $30 \%(\mathrm{w} / \mathrm{v})$ sucrose in deionized water. The brains were sectioned in the coronal plane at a thickness of $40 \mu \mathrm{m}$ on a freezing microtome and collected in three series. Nissl-stained sections were imaged to assess tissue damage (at $720 \mu \mathrm{m}$ intervals for PER lesions and $360 \mu \mathrm{m}$ intervals for POR lesions). Damage was identified by missing tissue, cell necrosis, or marked cortical thinning. The Cavalieri method was used to estimate lesion volume. Briefly, Cavalieri estimation is accomplished using a point grid with points spaced evenly along the $x$ and $y$ dimensions. The grid is placed randomly over a photomicrograph and points in the region of interest are counted. Area is calculated and multiplied by the distance between coronal sections to obtain volume. Points in the grid were spaced $250 \mu \mathrm{m}$ apart. For Experiment 2, lesion area was traced and quantified using Neurolucida software (MBF Bioscience) rather than the Cavalieri method. In cases of cortical thinning, the opposite hemisphere was drawn and used to estimate the extent of thinning. PER and POR borders were identified as described previously (Burwell, 2000). Due to the organization of intrinsic connections of the POR and PER (Burwell and Amaral, 1998), the amount of damage along the rostrocaudal extent of each region was assumed to be an important factor in the efficacy of the lesion. Therefore, the proportion of sections in the rostrocaudal plane that exhibited damage was quantified. A subject was retained in the study if the sections with damage were distributed across the rostrocaudal extent of the target region. In addition, we conducted Pearson correlation analysis to determine whether there was a relationship between lesion volume and discrimination performance.

\section{Statistical analysis}

FC tasks. Percentage freezing for each day of testing was analyzed by repeated-measures ANOVA (rANOVA) using "block" as the withinsubject variable and lesion "group" (sham, ipsilateral, contralateral) as the between-subject variable. For days that included tones or shocks, baseline freezing was analyzed separately from post-tone or post-shock epochs. Within-subject effects were analyzed by one-way ANOVA using "block" as the independent variable. Where sphericity was violated, as assessed by Maulchy's test of sphericity, the Greenhouse-Geisser correction was applied. It must be noted that, for the renewal FC, the first cohort to be conditioned (one rat from each lesion group) was removed from further analyses because experimental conditions differed. Our initial protocol called for $2 \mathrm{~s}$ foot shocks as described previously (Gershman et al., 2013). It became instantly apparent, however, that this was excessive as all three rats displayed agitated escape behavior rather than freezing during training and did not extinguish to tone in the following two sessions. For the remaining subjects, foot shock was reduced to $1 \mathrm{~s}$, which elicited normal freezing behavior. Importantly, excluding these subjects did not change significant statistical outcomes.

Object recognition tasks. Discrimination ratios were analyzed by rANOVA using "run" (days 1-8) as the within-subject variable and lesion "group" (sham, ipsilateral, contralateral) as the between-subject variable. Group effects were analyzed using planned comparisons between the contralateral group and either the sham or ipsilateral group. One-sample Student's $t$ tests were used to determine whether DRs differed significantly from zero. Bout numbers and bout durations were analyzed by rANOVA using "response" ( $\mathrm{N}$ or F) as the within-subject variable and lesion "group" as the between-subject variable. Within-subject effects were analyzed using independent samples Student's $t$ tests. Total exploration was analyzed by rANOVA using "run" and "phase" (S1, S2, T) as within-subject variables and lesion "group" as the between-subject variable. Within-subject effects were analyzed using independent-samples Student's $t$ tests.

\section{Results}

\section{Experiment 1}

We tested whether rats with crossed lesions of the PER and POR were impaired in two forms of contextual learning: FC and object recognition. Rats with contralateral $\mathrm{PER}-\mathrm{POR}$ lesions, ipsilateral PER-POR lesions, and sham-operated rats were tested in four tasks (Fig. 1). The first task was the unsignaled FC task, which assessed anterograde acquisition and expression of fear to a context. Inability to associate a mild foot shock (unconditioned stimulus) with context in this task would be evident during context extinction and would manifest as decreased freezing behavior. The second task was 3D cxtSOR, which tested the ability to recognize that an object has been placed in an incongruent context. Normal rats explore the novel object-context pairing preferentially and an impairment in this task would manifest as decreased exploration of the object in the incongruent context during the test phase. The third task was a standard, context-independent SOR control task was used to verify that any effects observed in the $3 \mathrm{D}$ cxtSOR were not due to impairments in object recognition per se. Therefore, all rats were expected to explore novel over familiar objects preferentially in the test phase. The final task was a tone-signaled ABBA renewal paradigm (renewal FC), which assessed renewal and retrieval of contextual fear. Impaired renewal to contextual cues would be evident on the fourth day and would manifest as decreased freezing during baseline (before tones are presented). Inability to use context to retrieve the original conditioned stimulus-unconditioned stimulus memory (i.e., the shock-tone in context A) would manifest as decreased freezing during post-tone epochs. We found that rats were impaired on 3D cxtSOR, but not on stdSOR. Rats were also unimpaired on context extinction during unsignaled FC and on context-dependent renewal after FC to a tone.

\section{Histology}

For all rats included in this study, obvious damage was distributed across the rostrocaudal extent of the target regions. The percentage (mean $\pm \mathrm{SE}$ ) of the sections that showed damage was $94 \pm 2$ in the ipsilateral group and $88 \pm 3$ in the contralateral group. The percentage of the volume damaged in the ipsilateral group was $65 \pm 5$ (PER), $75 \pm 6$ (POR), and $70 \pm 4$ (combined). The percentage of the volume damaged in the contralateral group was $70 \pm 6$ (PER), $71 \pm 7$ (POR), and $71 \pm 6$ (combined). Volume of damage was not significantly correlated with discrimination in the ipsilateral group ( $p$-values ranged from 0.41 to 0.83 ). In the contralateral group, discrimination performance was significantly negatively correlated with the volume of PER damage $(r=-0.83, p=0.04)$, but not POR damage $(p=0.32)$ or combined damage $(p=0.09)$. For the contralateral group, all correlations were negative, with $r$-values ranging from -0.49 to -0.83 . All PER lesions consistently targeted both areas 36 and 35 (Fig. 3). Most rats exhibited unilateral damage to cortical areas outside of the PER and POR (Fig. 3), but bilateral damage to the same extratarget area was not observed. In five rats with con- 

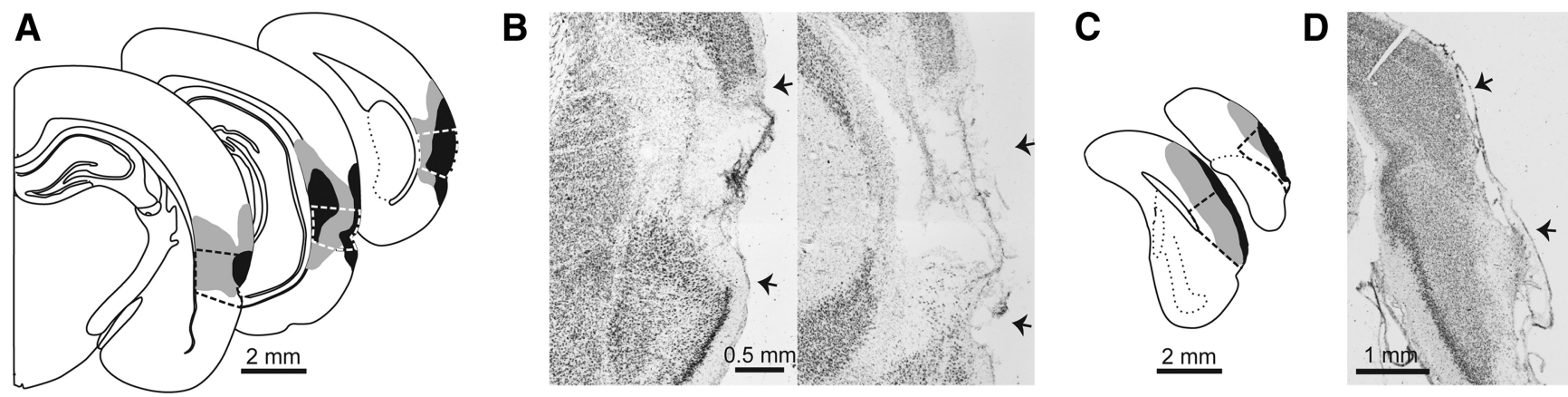

Figure 3. Lesion placement for Experiment 1. Schematic drawings show the smallest (black) and largest (gray) neurotoxic lesion for the PER ( $\boldsymbol{A}$ ) and POR ( $\boldsymbol{C}$. Contours are displayed on standard coronal sections at $-3.00,-5.04,-7.20 \mathrm{~mm}$ from bregma for PER and at -8.04 and $-9.12 \mathrm{~mm}$ for POR. Thick black or white dashed lines indicate area boundaries. Nissl staining of representative lesions are shown for PER at -3.36 and $-6.72 \mathrm{~mm}$ from bregma $(\boldsymbol{B})$ and POR at $-8.28 \mathrm{~mm}$ from bregma $(\boldsymbol{D})$. Arrows indicate area boundaries.

tralateral lesions, the POR lesion extended slightly into the caudal PER $(\sim 720 \pm 120 \mu \mathrm{m})$. In one rat, the PER lesion extended slightly into the rostral POR $(\sim 360 \mu \mathrm{m})$. Therefore, no rats were excluded from this study due to either excessive or insufficient damage.

\section{Contextual FC (unsignaled)}

Rats were fear conditioned to context on day 1 and then extinguished in the same context on day 2. During training, all rats demonstrated increased freezing behavior, as evidenced by an effect of "block" $\left(F_{(5,75)}=78.335, p=0.000\right)$ but not "block by group" ( $p=0.652$ ) during post-shock blocks (Fig. $4 A$, top). Baseline freezing during training did not differ across groups $(p=0.482)$. Rats with contralateral lesions to the PER-POR were not impaired in freezing to contextual cues during context extinction, as confirmed by rANOVA showing no main effect of "group" ( $p=0.989)$ and no "block by group" interaction ( $p=$ 0.847 ) on day 2 (Fig. $4 A$, bottom). There was a main effect of "block" $\left(F_{(6,90)}=2.636, p=0.021\right)$, which indicated that contextual freezing altered over the course of context extinction. Overall, our results show that contralateral damage to the PER-POR did not impair the formation of context-fear associations.

Tone-signaled ABBA renewal (renewal FC)

Rats in this task were fear conditioned to a tone in context $A$ on the first day, extinguished to tone in context $\mathrm{B}$ on the second and third days, and then tested for renewal in the original context $\mathrm{A}$ on the fourth day. We used baseline freezing to assess fear renewal in response to context $\mathrm{A}$ and post-tone freezing to assess contextguided retrieval of the original fear-tone association. As seen in Figure $4 B$, rats with contralateral lesions to the PER-POR were not impaired in either renewal or retrieval compared with the sham or ipsilateral groups. On day 4 (Fig. $4 B$, last panel), baseline freezing showed no main effect of "group" ( $p=0.094)$ and no "block by group" interaction $(p=0.176)$. A main effect of "block" $\left(F_{(1.38,16.61)}=6.737, p=0.013\right)$ provided evidence for renewal. There were no group differences during training or extinction (Fig. 4B, top panels and bottom left); on days 1,2 , or 3 , there were no effects of "group" for baseline freezing $(p=0.357,0.345$, $0.742)$, or for post-tone freezing $(p=0.168,0.290,0.354)$. No effects of "block by group" were observed in any of these periods. All rats were extinguished to tone, as evidenced by an effect of "block" on day 3 for post-tone freezing $\left(F_{(8,96)}=3.029, p=\right.$ $0.004)$. Overall, our results show that contralateral damage to the PER-POR did not impair context-guided renewal or retrieval.

$3 D$ cxtSOR

As expected, rats in the sham group explored object $\mathrm{N}$ preferentially over object $\mathrm{F}$ in the test phase (Fig. $4 C$, left). In contrast, rats in the contralateral group spent approximately the same amount of time exploring each object. Rats in the ipsilateral group tended to explore $\mathrm{N}$ more than $\mathrm{F}$, but this difference was not significant. This is evidenced by mean DR values that were significantly different from zero for the sham group ( $p=0.006, n=6)$, but not for the contralateral group ( $p=0.264, n=6)$ or the ipsilateral group ( $p=0.847, n=6$ ). Rats in the contralateral group were impaired compared with the sham group, as revealed by significantly reduced DR values compared with the sham $\left(F_{(1,10)}=\right.$ 5.91; $p=0.035)$, but not compared with the ipsilateral group $\left(F_{(1,10)}=1.07 ; p=0.325\right)$. There was no overall effect of "run" $(p=0.331)$ or "run by group" $(p=0.232)$ and no bias for side or objects was observed within or across subjects.

Analysis of exploration bouts in the test phase revealed that all rats explored $\mathrm{N}$ as frequently as $\mathrm{F}$ (Fig. $4 C$, middle). This was verified by an overall rANOVA showing no main effect of "group" ( $p=0.667)$, "response" $(p=0.332)$, or "response by group" ( $p=$ 0.981 ) for bout number. Bout durations for $\mathrm{N}$ versus $\mathrm{F}$, however, varied by group (Fig. $4 C$, right). For the sham group, $\mathrm{N}$ bouts tended to be longer than $\mathrm{F}$ bouts $(p=0.053)$, whereas for the contralateral and ipsilateral groups, bout durations were approximately the same for $\mathrm{N}$ and $\mathrm{F}$. The difference in trends between the sham and contralateral groups was statistically significant, as evidenced by an effect of "response by group" in an overall rANOVA $\left(F_{(1,10)}=7.44 ; p=0.021\right)$ and in a planned comparison between the sham and contralateral groups $\left(F_{(2,15)}=4.22 ; p=\right.$ $0.035)$. Total exploration time (all phases) did not differ across groups $(p=0.892)$ and mean exploration time was $14.19 \pm$ 0.34 s. All rats explored more during the sample phases (15.32 \pm $0.41 \mathrm{~s})$ compared with the test $(11.83 \pm 0.58 \mathrm{~s} ; p=0.000, n=$ 225 ), an unsurprising result considering overall novelty is less salient in the test phase. All rats explored more during the first 4 runs $(16.02 \pm 0.48 \mathrm{~s})$ than during the last $4(12.36 \pm 0.47 \mathrm{~s} ; p=$ 0.000 ), reflecting habituation for the task in general. Overall, our results show that rats with sham lesions explored object $\mathrm{N}$, a familiar object presented in an incongruent context, preferentially, whereas rats with contralateral lesions explored both objects equally. This indicates that rats with crossed PER-POR lesions failed to recognize novelty in the incongruent objectcontext pairing.

\section{$3 D$ stdSOR}

This task was performed to ensure that any impairment observed in the 3D cxtSOR was caused by an inability to discriminate novel object-context combinations and not by an inability to recognize the objects themselves. As expected, all rats explored the novel object preferentially in the test phase (Fig. $4 D$, left). The mean DR for the sham $(p=0.029, n=6)$, ipsilateral $(p=0.006, n=6)$, 
A
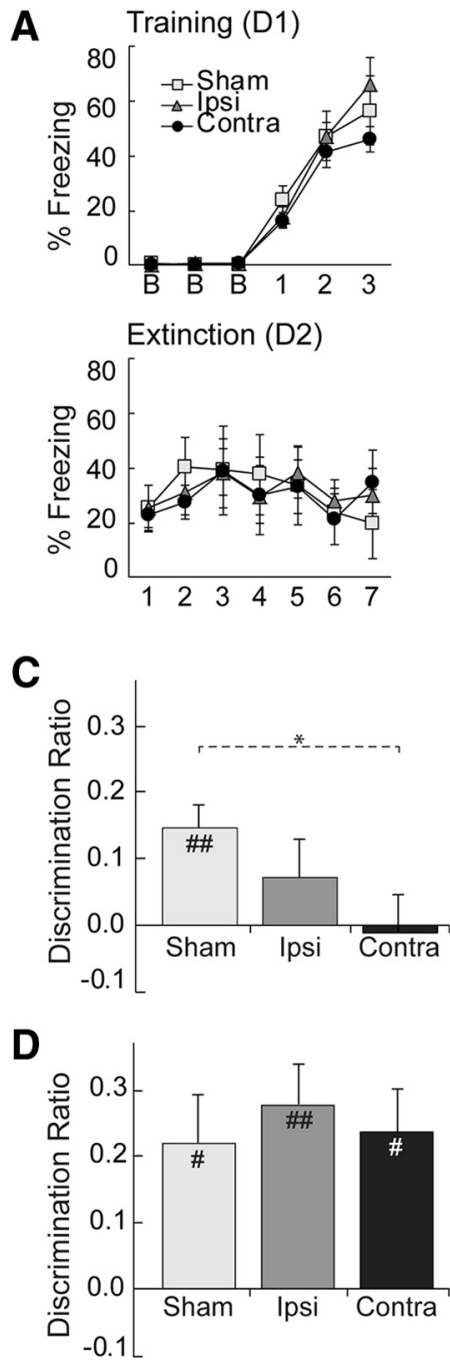
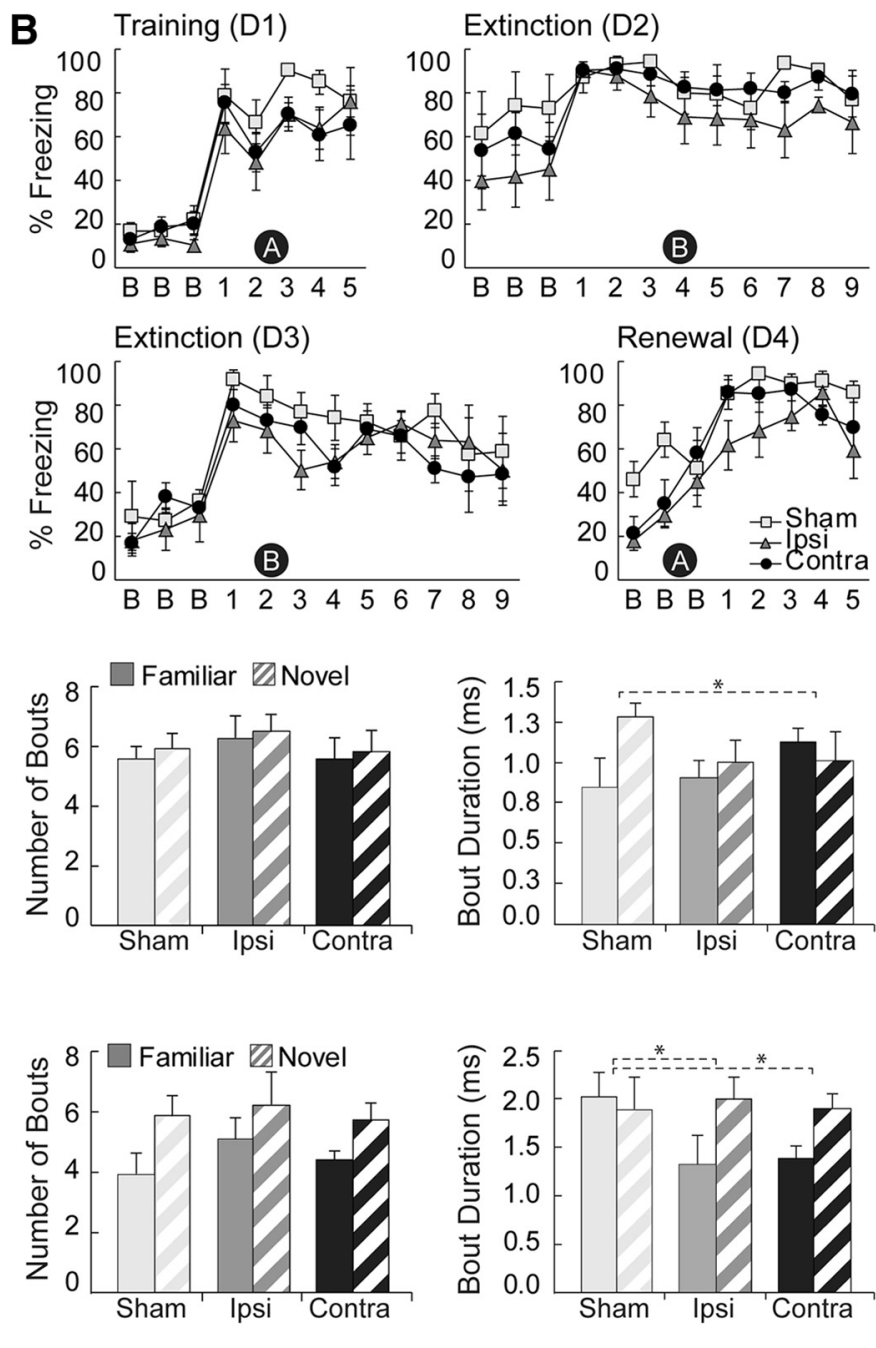

Figure 4. Behavioral results for Experiment 1. A, Percentage freezing in the unsignaled FC task during training and context extinction. There were no group differences in post-shock freezing or freezing during context extinction. $\boldsymbol{B}$, Exploration for the test phase of the 3D cxtSOR task. Both objects were familiar, but one was presented in an incongruent context (N) and the other in a congruent context (F). Shown are the discrimination ratio [left, $D R=(N-F) /(N+F)$, number of exploratory bouts (middle), and duration of exploratory bouts (right). The sham and ipsilateral groups showed discrimination, but the contralateral group did not. All groups showed a greater number of exploration bouts for the novel object in context. Sham and contralateral groups differed in exploratory bout duration such that only the sham group showed the expected increased bout duration for the novel object in context. $\boldsymbol{C}$, Exploration during the test phase of the standard SOR task. At test, one object is novel and the other is familiar. Panel order is identical to that in $\boldsymbol{B}$. As expected, all three groups exhibited a positive discrimination value and a greater number of exploratory bouts for the novel object. The sham group did not show the expected increased bout duration for the novel object. $\boldsymbol{D}$, Percentage freezing in the ABBA renewal FC task during training, tone extinction, and context extinction. Black circles with a white letter denote context, which was defined using visual, olfactory, auditory, extramaze, and tactile cues. Data are means $\pm \mathbf{S E M}$. In $\boldsymbol{A}-\boldsymbol{C}$, sample sizes were $n=6 /$ group and, in $\boldsymbol{D}$, sample sizes were $n=5 /$ group. For $\boldsymbol{B}$ and $\boldsymbol{C}$, $t$ test differences from zero were as follows: \#p $<0.05$, \#\#\# $p<0.001$; rAN0VA: ${ }^{*} p<0.05,{ }^{* * *} p<0.001$. D1-D4, Days 1-4, respectively.

and contralateral ( $p=0.016, n=6$ ) groups were significantly different from zero and an overall rANOVA showed no effect of "group" ( $p=0.823)$, "run” ( $p=0.565)$, or "run by group" $(p=$ 0.812).

As expected, bout numbers for all rats were more frequent for object $\mathrm{N}$ than for object $\mathrm{F}$ (Fig. $4 D$, middle), as evidenced by a "response" effect $\left(F_{(1,15)}=22.35 ; p=0.000\right)$ and no effects of "group" ( $p=0.692)$ or "response by group" $(p=0.583)$. Bout durations for $\mathrm{N}$ versus $\mathrm{F}$ varied by group (Fig. $4 D$, right), as evidenced by an overall effect of "response by group" $\left(F_{(2,15)}=4.86 ; p=\right.$ $0.024)$. Planned comparisons revealed a significant "response by group" effect for the sham versus ipsilateral $\left(F_{(1,10)}=7.295 ; p=\right.$ $0.022)$ and sham versus contralateral $\left(F_{(1,10)}=5.46 p=0.042\right)$ groups, but not for the ipsilateral versus contralateral groups $(p=0.534)$. Total exploration time did not differ across groups $(p=0.173)$; mean exploration time was $15.09 \pm 0.05 \mathrm{~s}$ and all rats explored equally across phases and runs. Overall, our results show that all rats explored the novel object preferentially over the familiar, indicating that they were able to recognize object novelty.

\section{Experiment 2}

We tested a new cohort of rats on two additional context-guided tasks (Fig. 2). First, signaled FC was used to further assess whether the PER-POR disconnection affects anterograde acquisition and expression of fear to context. This paradigm served to rule out the possibility that PER-POR disconnection might have a different effect on contextual FC in the presence of an explicit tone-shock association. In the second task, we tested the impact of the PERPOR disconnection on recognition of novel object-context combinations on a cxtSOR task that relies exclusively on $2 \mathrm{D}$ visual cues (2D cxtSOR) for both context and objects. We expected 

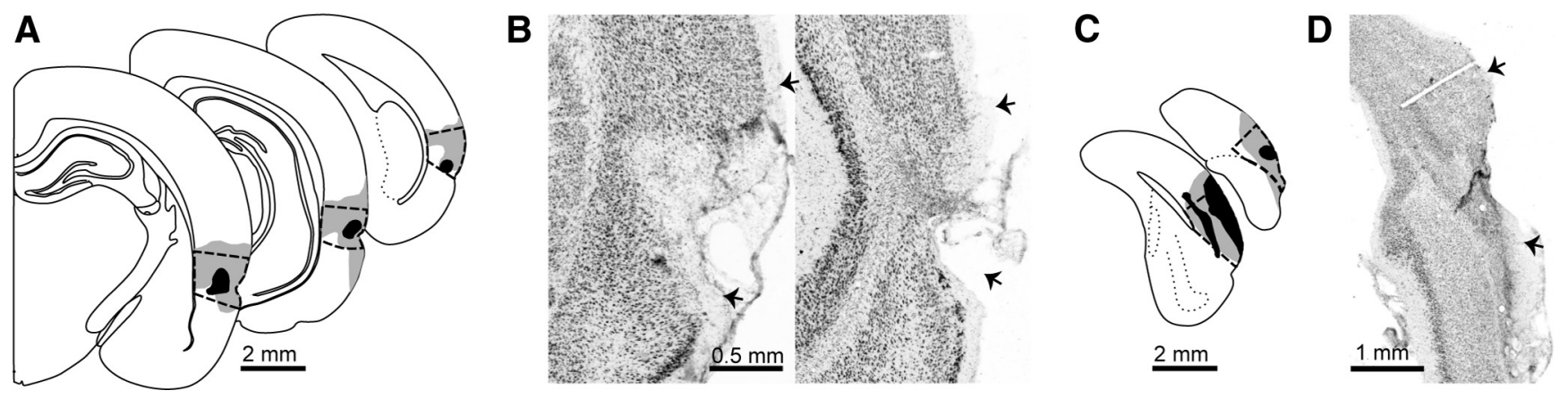

Figure 5. Lesion placement for Experiment 2. Schematic drawings show the smallest (black) and largest (gray) neurotoxic lesion for the PER ( $\boldsymbol{A}$ ) and POR (C). Contours are displayed on standard coronal sections at $-3.00,-5.04,-7.20 \mathrm{~mm}$ from bregma for PER and at -8.04 and $-9.12 \mathrm{~mm}$ for POR. Thick black or white dashed lines indicate area boundaries. Nissl staining of representative lesions are shown for PER at -3.36 and $-6.72 \mathrm{~mm}$ from bregma $(\boldsymbol{B})$ and POR at $-8.28 \mathrm{~mm}$ from bregma (D). Arrows indicate area boundaries.
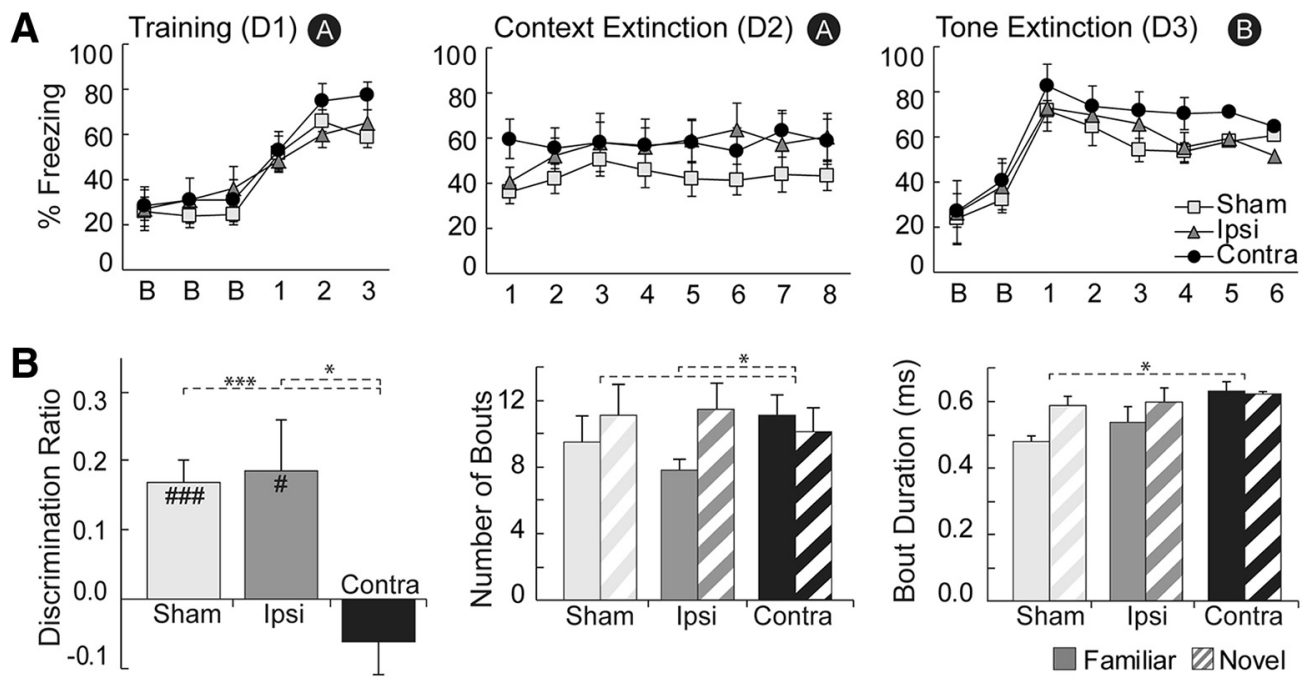

Figure 6. Behavioral results for Experiment 2. A, Percentage freezing in the signaled $\mathrm{FC}$ task during training in context $\mathrm{A}$, context extinction in context $\mathrm{A}$, and tone extinction in context $\mathrm{B}$. There were no group differences in any phase of the task. $\boldsymbol{B}$, Exploration during the test phase of the $2 \mathrm{D}$ cxtSOR task. Both objects were familiar, but one was presented in an incongruent context (N) and the other in a congruent context (F). Shown are the discrimination ratio [left, $D R=(N-F) /(N+F)]$, number of exploratory bouts (middle), and duration of exploratory bouts (right). The sham and ipsilateral groups showed discrimination, but the contralateral group did not. The sham and ipsilateral groups, but not the contralateral group, showed a greater number of exploration bouts for the novel object in context. The sham and contralateral groups also showed the expected longer duration of exploratory bouts. $t$ test differences from zero were as follows: \#p $<0.05$, \#\#\# $<0.001$; rANOVA: ${ }^{*} p<0.05,{ }^{* * *} p<0.001$. Error bars indicate \pm SEM. Sample sizes for the sham, ipsilateral (ipsi), and contralateral (contra) groups were $n=8,7$, and 7, respectively.

PER-POR disconnection to have similar effects on 2D cxtSOR as we had observed for 3D cxtSOR in Experiment 1.

\section{Histology}

For all rats included in this study, damage was distributed along the rostrocaudal extent of the target regions. The percentage (mean $\pm \mathrm{SE}$ ) of sections that showed damage was $88 \pm 5$ for the ipsilateral group and $86 \pm 4$ for the contralateral group. The percentage of the volume damaged in the ipsilateral group was $34 \pm 9$ (PER), $60 \pm 11$ (POR), and $47 \pm 8$ (combined). The percentage of the volume damaged in the contralateral group was $33 \pm 9$ (PER), $50 \pm 11$ (POR), and $41 \pm 9$ (combined). In the ipsilateral group, discrimination performance was significantly correlated with the volume of PER damage $(r=-0.83, p=0.04)$, but not POR damage ( $p=0.78)$ or combined damage $(p=0.20)$. Volume of damage was not significantly correlated with discrimination in the contralateral group (PER, $p=0.15$; POR, $p=0.49$; combined, $p=0.29)$. All six correlations were negative, with $r$-values ranging from -0.31 to -0.90 . Lesions in all but two rats were very well distributed along the rostrocaudal extent of the target regions (Fig. 5). In one rat with an ipsilateral lesion, there was minor sparing of rostral POR and, in one rat with a contralat- eral lesion, there was minor sparing of rostral PER. All PER lesions consistently targeted area 36 , whereas area 35 was partially spared in some rats. Some rats exhibited unilateral damage to cortical areas outside of the PER and POR (Fig. 5), but no rats exhibited bilateral damage within or outside of the target regions. Therefore, no rats were excluded from this study due to excessive or insufficient damage.

\section{Contextual FC (signaled)}

Rats were conditioned to a tone in context A on the first day, extinguished to context in the absence of tone on the second day, and then extinguished to tone in a new context on the third day. Rats with contralateral lesions to the PER-POR were not impaired in freezing to contextual cues (Fig. 6A), as confirmed by rANOVA showing no main effect of "group" $\left(F_{(2,19)}=1.102 ; p=\right.$ $0.353)$ or "block by group" $\left(F_{(8.4,79.4)}=0.983 ; p=0.457\right)$ on day 2. There was also no effect of block on day $2\left(F_{(4.2,79.4)}=1.816\right.$; $p=0.191)$. During training, baseline freezing did not differ across groups $\left(F_{(2,19)}=0.228 ; p=0.798\right)$ and all rats demonstrated increased freezing behavior during the post-shock blocks, as evidenced by an effect of "block" $\left(F_{(1.4,27.0)}=11.255 ; p=\right.$ $0.001)$, but not "block by group" $\left(F_{(2.8,27.0)}=1.082 ; p=0.371\right)$. 
As expected, all rats were extinguished to tone, as evidenced by an effect of "block" on day 3 during tone blocks $\left(F_{(2.6,50.0)}=5.821\right.$; $p=0.003)$, but not of "group" $\left(F_{(219)}=0.628 ; p=0.544\right)$ or "block by group" $\left(F_{(5.3,50.0)}=0.711 ; p=0.625\right)$. Overall, our results show that contralateral damage to the PER-POR did not impair the formation of context-fear or tone-fear associations.

Object in context recognition memory ( $2 \mathrm{D}$ cxtSOR)

As expected, rats in the sham and ipsilateral group explored object $\mathrm{N}$ preferentially over object $\mathrm{F}$, whereas rats in the contralateral group spent about the same amount of time exploring each object (Fig. 6B, left). This is demonstrated by mean DR values that were significantly different from zero for the sham group $(p=0.001, n=8)$ and the ipsilateral group $(p=0.042, n=7)$, but not for the contralateral group ( $p=0.252, n=7)$. Rats in the contralateral group were impaired compared with both the sham and ipsilateral group, as revealed by a significant group effect $\left(F_{(2,19)}=6.91 ; p=0.006\right)$ and by planned comparisons showing that DR values for the contralateral group were significantly reduced compared with both the sham group $\left(F_{(1,13)}=16.51 ; p=\right.$ $0.001)$ and the ipsilateral group $\left(F_{(1,12)}=8.14 ; p=0.015\right)$. There was no overall effect of "run" $(p=0.822)$ or "run by group" $(p=$ 0.941 ) and no preference for side or object was observed within or across subjects. These results were mostly consistent with the 3D cxtSOR except that, in this case, the ipsilateral group discriminated as expected.

The number of exploration bouts for $\mathrm{N}$ versus $\mathrm{F}$ varied by group; the sham and ipsilateral groups tended to explore object $\mathrm{N}$ more often than object F, whereas the contralateral group explored the two objects approximately equally (Fig. $6 B$, middle). The difference in these trends was statistically significant. This was evidenced by an effect of "response by group" $\left(F_{(2,19)}=4.486 ; p=\right.$ $0.025)$ in an overall rANOVA and in planned comparisons for the contralateral versus sham $\left(F_{(1,13)}=8.067 ; p=0.014\right)$ and contralateral versus ipsilateral $\left(F_{(1,12)}=6.872 ; p=0.022\right)$, but not sham versus ipsilateral groups $(p=0.3)$. Further analysis revealed that bout durations were longer when subjects in the sham group explored object $\mathrm{N}$ compared with object F ( $p=0.006, n=8)$, whereas the contralateral $(p=0.326)$ and ipsilateral $(p=0.904)$ groups explored both equally (Fig. $6 B$, right). The difference in trends between the sham and contralateral groups was marginally significant $\left(F_{(1,13)}=4.254 ; p=0.06\right)$.

Finally, total exploration times (all phases) did not vary across groups, as indicated by an overall rANOVA $(p=0.511)$, and the mean exploration time was $11.79 \pm 0.20 \mathrm{~s}$. There was an effect of phase $\left(F_{(2,38)}=3.816 ; p=0.031\right)$, but not of "phase by group" $(p=0.769)$, "run" $(p=0.193)$, or "run by group" $(p=0.252)$. As in the 3D cxtSOR, total exploration for all rats was significantly lower in the test phase $(11.12 \pm 0.37 \mathrm{~s})$ compared with the samples $(12.10 \pm 0.24 \mathrm{~s} ; p=0.027, n=88)$. Overall, our results show that rats with sham or ipsilateral lesions explored object $\mathrm{N}$ preferentially, a familiar object presented in an incongruent context, over object F, a familiar object presented in the same context in which it was encountered previously, whereas the contralateral group explored both objects equally. This indicates that rats with crossed PER-POR lesions failed to recognize novelty in the incongruent object-context pairing.

\section{Discussion}

Several paradigms that involve processing of context are impaired by damage to either POR or PER and available evidence suggests that the POR and the primate PHC are involved in representing context. Whether these two structures interact directly to encode contextual representations, however, is an open question. In the present study, we used a disconnection approach to determine whether the PER-POR interaction is necessary for context-guided behavior. In Experiment 1, we tested rats in three paradigms that required processing of environmental context, including 3D cxtSOR, unsignaled FC, and signaled FC followed by tone extinction and context-dependent renewal. Impairment in these tasks would indicate that communication between PER and POR is necessary for contextual learning in general. We also tested rats in standard SOR as a control task that does not rely on processing of context. As predicted, we found that rats were impaired on 3D cxtSOR and unimpaired on the stdSOR. Surprisingly, rats were unimpaired on context extinction during unsignaled FC and on context-dependent renewal after FC to a tone.

Experiment 2 further tested whether rats with crossed PERPOR lesions were impaired in recognizing novel object-context pairings and unimpaired in contextual FC. Because it is possible that context is processed differently in unsignaled and signaled FC paradigms, we assessed the effects of crossed PER-POR lesions on signaled FC. Indeed, dorsal hippocampal lesions have been shown to disrupt contextual fear acquisition in a signaled, but not in an unsignaled, procedure (Phillips and LeDoux, 1994). Because unimodal auditory stimuli can be processed directly via a thalamo-amygdalar route (Romanski and LeDoux, 1992a, 1992b), tone extinction served as another control ensuring that PERPOR damage did not disrupt processing of unimodal stimuli. In Experiment 2, the cxtSOR task used 2D visual cues exclusively for both context and objects. Because the use of $2 \mathrm{D}$ images in a noncontextual SOR task produces preferential novelty exploration comparable to those observed with 3D objects (Brown et al., 2010; Ho et al., 2015), we expected to replicate the 3D cxtSOR results. Replicating and extending the findings of Experiment 1, we found that rats were impaired on $2 \mathrm{D}$ cxtSOR and unimpaired in signaled FC.

To summarize, we showed that PER-POR disconnection impairs context-guided object recognition whether stimuli are multimodal or purely visual while sparing standard novel object recognition. In contrast $\mathrm{PER}-\mathrm{POR}$ disconnection has no impact on unsignaled or signaled FC or on context-dependent renewal after tone-shock conditioning. These findings suggest that, depending on how context is to be used, different or alternative circuits can support the processing of contextual information. PER-POR interaction is necessary for spontaneous contextguided exploration of novelty and unnecessary for associating context with a fear-producing stimulus. One possibility is that PER-POR interaction is normally involved in contextual FC, but in its absence, the hippocampus is able to encode information sufficient to retrieve fear memories.

The current findings provide the first evidence that PER and POR interaction is necessary for recognizing familiar objects in noncongruent but familiar contexts. What might be the basis for these impairments? Because disconnection does not impair object recognition per se, these impairments likely reflect an inability to either encode and discriminate between contexts or bind objects with a particular context. If rats with contralateral lesions regarded both objects as familiar without consideration for context, then one might expect decreased exploration times compared with the other two groups in the test phase. Analysis of exploration, however, revealed no group differences in the test phase, suggesting that rats in the contralateral group experienced novelty of some sort. Because PER-POR disconnection does not impair the ability to recognize objects, it is reasonable to posit 
that the cross-lesioned rats were not able to encode context during sample phases and/or recognize context during test.

Why might representations of context rely on PER-POR connectivity? Most environmental contexts are not simply places. Rather, contexts are characterized by a variety of features including the spatial layout of objects, items, and patterns in that place. Our results are consistent with an earlier finding that POR neurons in rats signal object-location conjunctions (Furtak et al., 2012). Likewise, PHC neurons in monkeys are responsive to both spatial and nonspatial stimuli (Sato and Nakamura, 2003). Human imaging studies show repetition suppression in PHC in response to simulated contexts (Szpunar et al., 2014) and higher PHC activity in response to novel object-context associations (Rémy et al., 2014). POR neurons recorded in a context-guided discrimination task showed object-context conjunctive coding for 2D objects embedded in patterned floors (Heimer-McGinn et al., 2016). We reported previously that local field potentials in POR exhibit strong power in the theta-frequency band, that theta in the POR is modulated by task demands, and that a large proportion of POR cells are phase locked to theta (Furtak et al., 2012). Other data suggest that theta coherence between the POR and PER may increase when rats are looking at a 2D image (Tomás Pereira and Burwell, 2014). Together with these studies, our findings support the view that the POR has a role in representing context and that object information, which is necessary for detailed context representations, may be received directly from the PER.

Although the notion that spatial and nonspatial input pathways to the hippocampus are segregated is a prominent one, an emerging view is that structures in the medial temporal lobe other than the hippocampus also link objects to locations (Eichenbaum et al., 2012; Knierim, 2015; Eichenbaum, 2017). This emerging view is consistent with our proposal that the PER provides object information to both the POR and to the hippocampus, but for different purposes. The PER provides object, item, and pattern information to the POR for representing the spatial layout of objects and patterns in the local environmental context. The PER provides this information to the hippocampus for associative learning, for example, when a particular object is rewarded in one context but not another. Indeed, a number of studies have reported that the hippocampus responds to objects as well as places, contexts, and landmarks (Komorowski et al., 2009; Deshmukh and Knierim, 2013; Scaplen et al., 2014).

It is important to acknowledge that PER-POR connections are reciprocal (Burwell and Amaral, 1998). If PER information in the POR is used for representing context, what is the purpose of the return projection? It is the case that object-location correlates have been observed in PER neurons in discrimination tasks in which the location of the object was relevant to the task (Ahn and Lee, 2015; Keene et al., 2016). In these studies, the location of the object determines whether it is rewarded. One possibility is that, in such tasks, the PER requires POR information to disambiguate the reward properties of an object. In other words, the locationdetermined reward properties are bound to the object representations. More specific targeting of the reciprocal PER-POR pathways will be necessary to clarify the purpose of POR information in the PER, as well as the purpose of PER information in the POR. What is clear from the present study, however, is that PER and POR connectivity is necessary for context-guided exploratory behavior.

Given the emerging importance of the PER-POR pathway in representing context, why is contextual FC not disrupted by crossed PER-POR lesions? Our results were surprising in light of prior work demonstrating the critical role that these cortical areas individually play in contextual FC and context-guided object recognition. For example, Bucci et al. (2000) showed that PER and POR are each essential for the acquisition and expression of context-fear associations, Burwell et al. (2004) demonstrated that both areas are necessary for remote memory of contextual fear, and Norman and Eacott (2005) showed that both areas are necessary for context-guided object recognition. The hippocampus is also implicated in the acquisition of contextual fear, although this contribution is sensitive to type of damage, timing of damage, and behavioral procedure (Anagnostaras et al., 2001; Maren, 2001; Sanders et al., 2003; Rudy et al., 2004). The most straightforward explanation for our findings is that the POR and PER rely on each other to form detailed representations of context that include the spatial layout of items and features contained within the context. The hippocampus normally relies on representations of context from the POR, but in the absence of refined context representations, it is able to support associative learning in contextual FC based on rudimentary representations of context from the POR. Alternatively, the hippocampus may associate other available features of the operant chamber with foot shock that are sufficient to retrieve conditioned fear. If a more complex representation of context is necessary, however, we would predict that the POR would require input from the PER.

To conclude, the results of this study show that contextguided exploratory behavior requires direct PER-POR interaction and that ipsilateral PER and POR input to the hippocampus is not sufficient. Although the relationship between function and directionality remains to be elucidated, these data are consistent with our hypothesis that the POR relies on object information from the PER to form complex representations of the local environmental context. Object-location conjunctions have been identified in the POR, PER, and hippocampus (Komorowski et al., 2009; Furtak et al., 2012; Keene et al., 2016). We propose that the PER, which projects directly to the POR and to the CA1 field of the hippocampus (Burwell and Amaral, 1998; Agster and Burwell, 2013), transmits object information to both structures, but for different purposes. That is, the POR uses object information from the PER for binding the spatial layout of objects and features in the local environment to form a complex representation of spatial context. Object information arriving to the hippocampus from the PER could be used for associative learning, for example, binding a particular event or item with the representation of environmental context provided by the POR. Our findings support this view by providing the first evidence that direct interaction between the PER and POR is necessary for context-guided exploratory behavior.

\section{References}

Agster KL, Burwell RD (2013) Hippocampal and subicular efferents and afferents of the perirhinal, postrhinal, and entorhinal cortices of the rat. Behav Brain Res 254:50-64.

Aguirre GK, D’Esposito M (1999) Topographical disorientation: a synthesis and taxonomy. Brain 122:1613-1628.

Ahn JR, Lee I (2015) Neural correlates of object-associated choice behavior in the perirhinal cortex of rats. J Neurosci 35:1692-1705.

Aminoff EM, Kveraga K, Bar M (2013) The role of the parahippocampal cortex in cognition. Trends Cogn Sci 17:379-390.

Anagnostaras SG, Gale GD, Fanselow MS (2001) Hippocampus and contextual fear conditioning: recent controversies and advances. Hippocampus 11:8-17.

Bachevalier J, Nemanic S, Alvarado MC (2015) The influence of context on recognition memory in monkeys: effects of hippocampal, parahippocampal and perirhinal lesions. Behav Brain Res 285:89-98. 
Bar M, Aminoff E (2003) Cortical analysis of visual context. Neuron 38:347-358.

Brown MW, Warburton EC, Aggleton JP (2010) Recognition memory: material, processes, and substrates. Hippocampus 20:1228-1244.

Bucci DJ, Phillips RG, Burwell RD (2000) Contributions of postrhinal and perirhinal cortex to contextual information processing. Behav Neurosci 114:882-894.

Bucci DJ, Saddoris MP, Burwell RD (2002) contextual fear discrimination is impaired by damage to the postrhinal or perirhinal cortex. Behav Neurosci 116:479-488.

Burgess N, Maguire EA, Spiers HJ, O'Keefe J (2001) A temporoparietal and prefrontal network for retrieving the spatial context of lifelike events. Neuroimage 14:439-453.

Burwell RD (2000) The parahippocampal region: corticocortical connectivity. Ann N Y Acad Sci 911:25-42.

Burwell RD, Amaral DG (1998) Perirhinal and postrhinal cortices of the rat: interconnectivity and connections with the entorhinal cortex. J Comp Neurol 391:293-321.

Burwell RD, Bucci DJ, Sanborn MR, Jutras MJ (2004) Perirhinal and postrhinal contributions to remote memory for context. J Neurosci 24:11023-11028.

Chrobak JJ, Amaral DG (2007) Entorhinal cortex of the monkey: VII. intrinsic connections. J Comp Neurol 500:612-633.

Corodimas KP, LeDoux JE (1995) Disruptive effects of posttraining perirhinal cortex lesions on conditioned fear: contributions of contextual cues. Behav Neurosci 109:613-619.

Deshmukh SS, Knierim JJ (2013) Influence of local objects on hippocampal representations: landmark vectors and memory. Hippocampus 23:253-267.

Dolorfo CL, Amaral DG (1998) Entorhinal cortex of the rat: organization of intrinsic connections. J Comp Neurol 398:49-82.

Eichenbaum H (2017) The role of the hippocampus in navigation is memory. J Neurophysiol 117:1785-1796.

Eichenbaum H, Yonelinas AP, Ranganath C (2007) The medial temporal lobe and recognition memory. Annu Rev Neurosci 30:123-152.

Eichenbaum H, Sauvage M, Fortin N, Komorowski R, Lipton P (2012) Towards a functional organization of episodic memory in the medial temporal lobe. Neurosci Biobehav Rev 36:1597-1608.

Ennaceur A, Delacour J (1988) A new one-trial test for neurobiological studies of memory in rats. 1: Behavioral data. Behav Brain Res 31:47-59.

Furtak SC, Ahmed OJ, Burwell RD (2012) Single neuron activity and theta modulation in postrhinal cortex during visual object discrimination. Neuron 76:976-988.

Gabrieli JD, Brewer JB, Desmond JE, Glover GH (1997) Separate neural bases of two fundamental memory processes in the human medial temporal lobe. Science 276:264-266.

Gaffan EA, Healey AN, Eacott MJ (2004) Objects and positions in visual scenes: effects of perirhinal and postrhinal cortex lesions in the rat. Behav Neurosci 118:992-1010.

Gershman SJ, Jones CE, Norman KA, Monfils MH, Niv Y (2013) Gradual extinction prevents the return of fear: implications for the discovery of state. Front Behav Neurosci 7:164.

Hayes SM, Nadel L, Ryan L (2007) The effect of scene context on episodic object recognition: parahippocampal cortex mediates memory encoding and retrieval success. Hippocampus 17:873-889.

Heimer-McGinn VR, Kent B, Burwell RD (2016) Representations of context in the postrhinal cortex. Program No. 84.03. 2016 Neuroscience Meeting Planner: San Diego, CA: Society for Neuroscience, 2016. Online.

Ho JW, Poeta DL, Jacobson TK, Zolnik TA, Neske GT, Connors BW, Burwell RD (2015) Bidirectional modulation of recognition memory. J Neurosci 35:13323-13335.

Jacobson TK, Ho JW, Kent BW, Yang FC, Burwell RD (2014) Automated visual cognitive tasks for recording neural activity using a floor projection maze. J Vis Exp 84:e51316.

Keene CS, Bladon J, McKenzie S, Liu CD, O’Keefe J, Eichenbaum H (2016) Complementary functional organization of neuronal activity patterns in the perirhinal, lateral entorhinal, and medial entorhinal cortices. J Neurosci 36:3660-3675.
Knierim JJ (2015) The hippocampus. Curr Biol 25:R1116-R1121.

Komorowski RW, Manns JR, Eichenbaum H (2009) Robust conjunctive item-place coding by hippocampal neurons parallels learning what happens where. J Neurosci 29:9918-9929.

Maguire EA, Frith CD, Burgess N, Donnett JG, O’Keefe J (1998) Knowing where things are parahippocampal involvement in encoding object locations in virtual large-scale space. J Cogn Neurosci 10:61-76.

Malkova L, Mishkin M (2003) One-trial memory for object-place associations after separate lesions of hippocampus and posterior parahippocampal region in the monkey. J Neurosci 23:1956-1965.

Maren S (2001) Neurobiology of Pavlovian fear conditioning. Annu Rev Neurosci 24:897-931.

Martin CB, McLean DA, O’Neil EB, Köhler S (2013) Distinct familiaritybased response patterns for faces and buildings in perirhinal and parahippocampal cortex. J Neurosci 33:10915-10923.

Mullally SL, Maguire EA (2011) A new role for the parahippocampal cortex in representing space. J Neurosci 31:7441-7449.

Norman G, Eacott MJ (2005) Dissociable effects of lesions to the perirhinal cortex and the postrhinal cortex on memory for context and objects in rats. Behav Neurosci 119:557-566.

Phillips RG, LeDoux JE (1994) Lesions of the dorsal hippocampal formation interfere with background but not foreground contextual fear conditioning. Learn Mem 1:34-44.

Ploner CJ, Gaymard BM, Rivaud-Péchoux S, Baulac M, Clémenceau S, Samson S, Pierrot-Deseilligny C (2000) Lesions affecting the parahippocampal cortex yield spatial memory deficits in humans. Cereb Cortex 10:1211-1216.

Ranganath C, Yonelinas AP, Cohen MX, Dy CJ, Tom SM, D’Esposito M (2004) Dissociable correlates of recollection and familiarity within the medial temporal lobes. Neuropsychologia 42:2-13.

Rémy F, Vayssière N, Pins D, Boucart M, Fabre-Thorpe M (2014) Incongruent object/context relationships in visual scenes: where are they processed in the brain? Brain Cogn 84:34-43.

Renner MJ, Seltzer CP (1991) Molar characteristics of exploratory and investigatory behavior in the rat (Rattus norvegicus). J Comp Psychol 105:326-339.

Romanski LM, LeDoux JE (1992a) Bilateral destruction of neocortical and perirhinal projection targets of the acoustic thalamus does not disrupt auditory fear conditioning. Neurosci Lett 142:228-232.

Romanski LM, LeDoux JE (1992b) Equipotentiality of thalamo-amygdala and thalamo-cortico-amygdala circuits in auditory fear conditioning. J Neurosci 12:4501-4509.

Rudy JW, Huff NC, Matus-Amat P (2004) Understanding contextual fear conditioning: insights from a two-process model. Neurosci Biobehav Rev 28:675-685.

Sanders MJ, Wiltgen BJ, Fanselow MS (2003) The place of the hippocampus in fear conditioning. Eur J Pharmacol 463:217-223.

Sato N, Nakamura K (2003) Visual response properties of neurons in the parahippocampal cortex of monkeys. J Neurophysiol 90:876-886.

Scaplen KM, Gulati AA, Heimer-McGinn VL, Burwell RD (2014) Objects and landmarks: hippocampal place cells respond differently to manipulations of visual cues depending on size, perspective, and experience. Hippocampus 24:1287-1299.

Suzuki WA, Amaral DG (1994) Topographic organization of the reciprocal connections between the monkey entorhinal cortex and the perirhinal and parahippocampal cortices. J Neurosci 14:1856-1877.

Szpunar KK, St Jacques PL, Robbins CA, Wig GS, Schacter DL (2014) Repetition-related reductions in neural activity reveal component processes of mental simulation. Soc Cogn Affect Neurosci 9:712-722.

Tomás Pereira I, Burwell R (2014) Neuronal activity in the perirhinal and postrhinal cortex during performance on a contextual spontaneous object recognition task. Program No. 176.07. 2014 Neuroscience Meeting Planner. Washington, D.C. Society for Neuroscience, 2014. Online.

Wilson DI, Langston RF, Schlesiger MI, Wagner M, Watanabe S, Ainge JA (2013) Lateral entorhinal cortex is critical for novel object-context recognition. Hippocampus 23:352-366. 